\title{
Lessons from the Department of Energy's Pandemic Response for Multidisciplinary Research
}

\author{
Julienne M. Krennrich \\ Director, Innovation Partnerships \\ jmkrenn@ameslab.gov \\ https://orcid.org/0000-0002-9683-838X \\ James R. Morris, Ames Laboratory \\ https://orcid.org/0000-0002-8464-9047
}

$\mathrm{O}$

ne of the hallmarks of science in the COVID-19 era is the remarkable advances that were made scientifically to address the pandemic. The rapid genomic analyses of the SARS-Cov-2 virus (and subsequent rapid sharing), the development and roll-out of vaccines, and other advances demonstrate both the ability to rapidly address new challenges and the ability to leverage strong research directions that have been in development for decades. However, the challenges were not simply in the areas of biology and medicine and are ongoing on multiple frontiers. From the experience of U.S. Department of Energy (DOE) labs, we see lessons to be learned in driving multi-institution, multidisciplinary research efforts that address significant challenges. While they were not set up specifically to address challenges such as this pandemic, their structure, organization, capabilities, and mission allowed them to pivot, dedicate significant resources, and rapidly form coherent research efforts across disciplines, capabilities, and institutions, to initiate and accomplish significant results in short times. The present paper describes the view from one of these laboratories, with the perspective of what may be learned toward organizing effective, larger research efforts.

\section{About Department of Energy Laboratories and Ames Laboratory \\ Ames Laboratory (Ames) is one of 17 national laboratories owned by DOE (see Fig. 1), and one of 10 operated by the DOE Office of Science.}

The laboratory is operated by the Iowa State University of Science and Technology (ISU) via a Management and Operating Contract (M\&O). This type of government contract is well-suited to managing risky work, such as research and development. This scenario is maximally beneficial for both Ames and ISU, enabling easy collaboration between ISU faculty and students and Ames scientists. Additionally, Ames benefits operationally from access to campus services and collaboration on emergency response and protective services.

One defining characteristic of the DOE Laboratories is their mission to lead large research and development ef- forts that require scale and provide user facilities to the scientific community. By combining state-of-the-art facilities with larger research programs, often spanning multiple institutions, including other DOE labs, universities, and industry, the Department of Energy tackles important scientific and technological challenges. For example, among a number of other projects, Ames leads the Critical Materials Institute ${ }^{1}$ (an Energy Innovation Hub spanning four DOE labs, 13 universities, and many industries), two Energy Frontier Research Centers (EFRC), ${ }^{2}$ and is a thrust leader for the Exascale Computing Project. ${ }^{3}$ These efforts tackle significant basic, applied, and computational challenges within the physical sciences and are coordinated across multiple laboratories. Much of Ames' funding comes from the Office of Science's Basic Energy Sciences (BES) program, which is one of the largest sponsors of research in the physi- 
Figure 1: A map of the U.S. Department of Energy Laboratories.

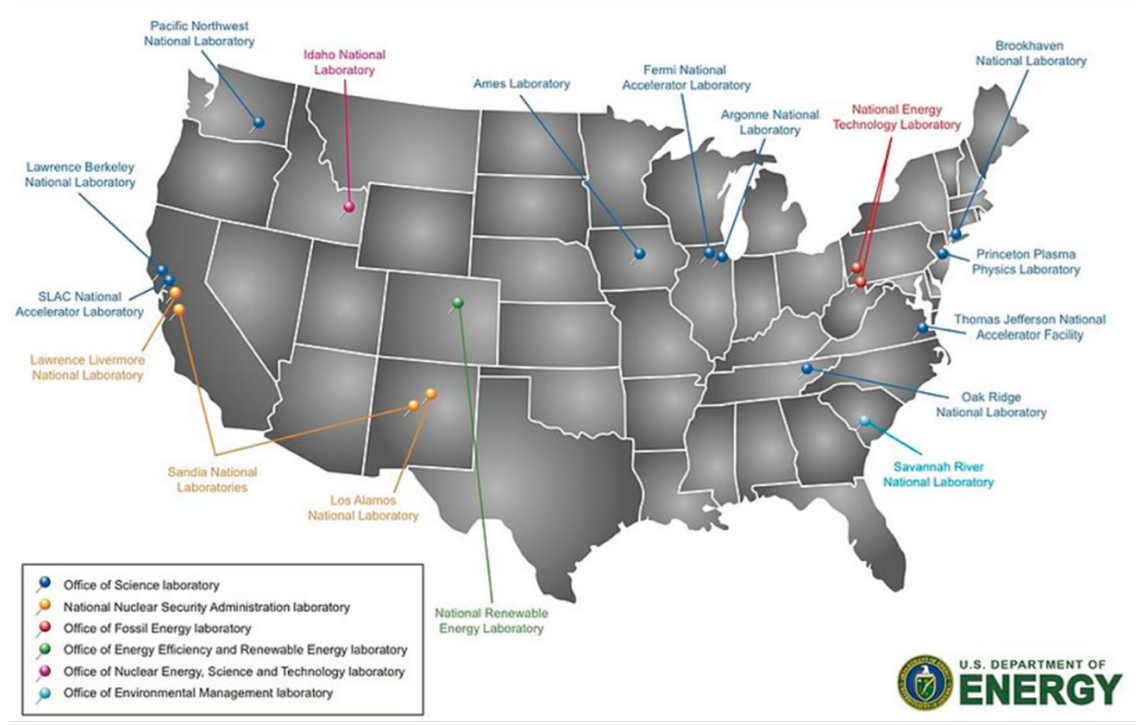

cal sciences and operates significant programs and facilities supporting the DOE mission. BES has had a significant impact since its inception. ${ }^{4}$

Response to COVID-19 Global Pandemic

When COVID-19 became a global pandemic in spring 2020, one of the big research challenges was coordinating across federal agencies to effectively address myriad response-related challenges. The U.S. Department of Health and Human Services and the National Institute of Allergy and Infectious Diseases were the epicenter of the disease expertise itself. The Federal Emergency Management Agency had been doing epidemic modeling before, but suddenly this was needed on a massive scale. One issue raised was how to combine epidemiological modeling with human behaviors and economic modeling. We knew that opening up businesses and schools would increase infection rates, but by how much? This more comprehensive modeling could really drive local policy on business/school opening, since different conditions and behaviors were highly dependent on local conditions.

There were also medical equipment supply chain issues. For example, where were the resources (masks, gloves, ventilators, tests) compared to where the needs were (which changed rapidly week to week, with little data on local resources)? At one point, every mechanical engineering department in the nation was suddenly designing ventilators; but how does one quickly sort through those to prioritize the testing needed for formal approval? Adding to the foray was the CARES Act ${ }^{5}$ pushing research dollars to tackle these challenges with very short turnarounds for impactful deliverables.

In response, DOE created the $\mathrm{Na}$ tional Virtual Biotechnology Laboratory (NVBL). ${ }^{6}$ The NVBL could be set up rapidly due to coordination between the labs, their existing collaborations and complementary strengths, and culture of collaborative, multi-institutional collaboration. The DOE's Office of Science leadership quickly established the framework for how the NVBL would operate. The NVBL was led by two co-chairs who provided oversight and organization of a central committee with representation from all 17 laboratories. This committee organized the research priorities into top-level challenges. Then, through their representatives, each lab had an opportunity to propose what they could contrib- 
ute to each challenge topic. The responses captured not only their capabilities and expertise, but also their ability to leverage existing partnerships. This accelerated the transition of the research into practice. The NVBL committee then collaboratively assembled these complementary "work statements" into projects under each topic area. Every project involved multiple laboratories and was selected according to the potential impact, the ability to coordinate while maintaining separate directions from other agencies.

The Ames Laboratory's role in the Viral Fate $\mathcal{E}$ Transport ${ }^{7}$ project is instructive as an example. Ames has significant expertise in the physics and chemistry of alloys and metal oxides. The ability to tap into ISU's expertise and its BSL3 facility was an additional asset we could contribute that helped speed up measurements on an actual virus. The Ames-ISU partnership, in conjunction with Sandia National Laboratories, led to the discovery of a new approach to designing antiviral materials with a low toxicity to humans. Similarly, Ames was able to tap the deep expertise of an Ames/ISU joint faculty member to tackle the need for rapid onsite testing for virus presence as part of the COVID-19 Testing R\&D. ${ }^{8}$

A key attribute that made the NVBL a success was the fact that the laboratories specialize in mission-oriented research that is responsive to dynamic priorities. They also specialize in tackling problems that require scale: research that takes advantage of numerous collaborative scientists working together with unique facilities (often larger-scale user facilities). Researchers in the laboratories are accustomed to working in multi-institutional teams combining multiple disciplines, as well as with university and industry partners, to identify and accomplish shared goals and challenges. Additionally, due to the M\&O model, DOE could quickly disseminate funds to the labs. Although the NVBL framework was the first of its kind, and undertaken in a "maximum telework" environment, it turned out to be relatively easy to stand up, due to the existing research culture.

The DOE utilized its expertise and multiple strengths to address the pandemic response, complementing and supporting other national agencies. The NVBL addressed medical equipment supply shortages, discovered potential drugs to fight the virus, developed and verified COVID-19 testing methods, modeled disease spread and impact across the nation, and worked to understand virus transport in buildings and the environment. National laboratory resources leveraged for this effort included a suite of world-leading user facilities broadly available to the research community, such as light and neutron sources, nanoscale science research centers, genomic sequencing and biocharacterization facilities, and high-performance computing facilities.

Legacy of the NVBL: How will it shape the future?

The pandemic response demonstrated DOE's ability to formulate "rapid response" groups of scientists that could devote significant resources and expertise to a mission-oriented (not academic-oriented) issue. Publications came but weren't the goal. These teams were able to demonstrate significant impact within a matter of months. For example, "within just a few months, NVBL teams produced innovations in materials and advanced manufacturing that mitigated shortages in test kits and personal protective equipment, creating nearly 1,000 new jobs." ${ }^{\prime 9}$ There has been ongoing discussion within DOE about creating a "Scientist Reserve Corps" that could mimic the NVBL type of response in preparation for future crises.

Within the context of climate change and the need to transition to a clean energy economy, the quick progress that has been made by the NVBL in transitioning $R \& D$ to commercial sector use really stands out as a possible model 
moving forward. We believe we will see more efforts like these in other mission areas. For example, DOE is now defining Energy Earthshots, such as the one on hydrogen. ${ }^{10}$ This is being coordinated by Science and Energy Tech Teams that cross DOE offices. DOE is also running more prize competitions. These represent areas where, with concentrated effort and resources, the research community really can make a difference in a short amount of time.

National challenges are inherently multidisciplinary, and rapid change requires more than technical solutions. The COVID-19 pandemic has demonstrated the importance of issues beyond science, such as scale up, supply chains, manufacturing (masks, tests), human behavior (willingness to wear personal protective equipment, isolate), policy (school, business shutdowns), and economics (business reopening).

In order to have a holistic response, the science had to be combined with all of the above to be successful. The key point is that the challenge drives the collaboration across fields. Articulating key issues carefully can be used to drive multi-institution/multidisciplinary collaborations.

Lessons for Multi-institution and Multidisciplinary Research

As we move into the future, we will confront Grand Challenges like $\mathrm{CO}_{2}$ reduction; electric and autonomous transport; data, AI and health care; and placebased issues of energy and water. We should expect to see an increased need for collaboration across not just disciplines and institutions but also technology scales, such as deep expertise in basic science, engineering, technology demonstration, technology transfer/partnerships and deployment. The DOE Labs are well positioned to work with our university and industry partners to tackle these challenges.

More broadly, we point out that there is a broader lesson for successful projects that span multiple disciplines and multi- ple institutions. Such interactions often struggle due to narrower interests of a particular research group, institution, or discipline. NVBL had success not by addressing (only) individual groups, but rather by identifying a few, well-articulated directions, with clear expectations for rapid progress and for collaborative work, and guiding the research along those directions. Having a clear mission that is compelling, important, and wholly bought into by all researchers is crucial. Prioritizing cross-disciplinary and/ or cross-institution efforts clearly also plays a role in forming such collaborative groups.

As an example of an important issue that undoubtedly spans multiple disciplines, from fundamental sciences to engineering to social sciences, consider the needs required to efficiently provide clean water across society. The issues are both local (what are local sources and conditions of water, what are local facilities or industries that require water, or that affect clean water supply) and non-local (local conditions are often strongly affected by what happens elsewhere; a community often will be strongly affected by what happens upstream). Increasing clean water supply may require more efficient approaches to remove contaminants, more efficient energy production (requiring less water resources), and new approaches to reduce sources of contamination (affecting industry and agriculture). Economic, infrastructure, and human behavior all directly impact options. While there are myriad research programs in each of these areas, we see potential for impactful research that combines multiple aspects, providing important demonstrations of the interplay and competition between these different areas.

Researchers require some independence and need to be able to bring forth ideas and act upon them, while balancing the importance of achieving the mission. The key motivating challenges of the research - rather than the individual disci- 
plines that may address them - are more likely to get researchers to work together than "blank slate" approaches to teaming. Focusing and refining (as needed) these challenges are more likely to create and maintain cross-disciplinary research. We also posit that this is key to impactful research: having multiple constituencies agreeing on important goals often produces research unique to the collaboration, with impacts that are easier to articulate due to the clear goals. Such collaborations are often hard to maintain, but good leaders recognize how the pieces serve a larger whole, and serve to both allow individual researchers some freedom to explore and to innovate, while keeping projects from devolving into handfuls of more parochial interests. To confront and respond to our pressing national challenges, the research culture needs to be collaborative, innovative, and nimble-rather than focused purely on small group/small project visions. The Department of Energy, and its National Laboratory system, has long sought to nurture this type of culture, balancing mission-focused work with core expertise and capabilities.

\section{References}

1. Critical Materials Institute. (2020). Annual Report. U.S. Department of Energy. https://iastate.box.com/v/cmi-annual-report

2. Energy Frontier Research Centers. U.S. Department of Energy. Retrieved August 13, 2021, from https://science.osti.gov/bes/efrc/Centers

3. Exascale Computing Project, Chemistry and Materials, GAMESS. U.S. Department of Energy. Retrieved August 13, 2021, from https://www. exascaleproject. org/research-group/chemistry-and-materials/

4. Drell, Persis, Bonnell, Dawn A., Chen, Jingguang, Clark, Sue, Cuenya, Beatriz Roldan, Dosch, Helmut, Friend, Cynthia, Gao, Yan, Hammes-Schiffer, Sharon, Kastner, Marc, Kay, Bruce, Leone, Stephen R., Louca, Despina, de la Cruz, Monica Olvera, Ourmazd, Abbas, Piot, Philippe, Robertson, Ian, Rollett, Anthony, Ross, Frances, Rubloff, Gary, Santore, Maria, Takeuchi, Esther S., Tranquada, John, \& Wasserman, Stephen. (2018, June 01). A Remarkable Return On Investment In Fundamental Research: 40 Years of Basic Energy Sciences at the Department of Energy. United States. https://doi.org/10.2172/1545686

5. The Coronavirus Aid, Relief, and Economic Security (CARES) Act. (2020, March 27). Retrieved September 1, 2021, from https://home.treasury.gov/policy-issues/ coronavirus/about-the-cares-act

6. National Virtual Biotechnology Laboratory. U.S. Department of Energy. https:// science.osti.gov/nvbl

7. National Virtual Biotechnology Laboratory. Viral Fate and Transport Highlights. U.S. Department of Energy. https://science.osti.gov/nvbl/NVBL-Projects/ViralFate-and-Transport

8. National Virtual Biotechnology Laboratory. COVID-19 Testing RED Highlights. U.S. Department of Energy. https://science.osti.gov/nvbl/NVBL-Projects/COVID19-Testing

9. National Virtual Biotechnology Laboratory. (2020). RED for Rapid Response to the COVID-19 Crisis. U.S. Department of Energy. p. 1. https://science.osti.gov/-/media/ nvbl/pdf/NVBL_Brochure Final_c.pdf

10. Hydrogen and Fuel Cell Technologies Office. Hydrogen Shot. U.S. Department of Energy. Retrieved August 13, 2021, from https://www.energy.gov/eere/fuelcells/ hydrogen-shot 